\title{
Multi-beam - enabling leading-edge mask writing (Conference Presentation) (Withdrawal Notice)
}

Christoph Spengler, Elmar Platzgummer, Hans Loeschner

Christoph Spengler, Elmar Platzgummer, Hans Loeschner, "Multi-beam enabling leading-edge mask writing (Conference Presentation) (Withdrawal Notice)," Proc. SPIE 10810, Photomask Technology 2018, 108100I (31 October 2018); doi: 10.1117/12.2503330

SPIE Event: SPIE Photomask Technology + Extreme Ultraviolet Lithography, 2018, Monterey, California, United States 


\section{Multi-beam - enabling leading-edge mask writing (Conference Presentation) (Withdrawal Notice)}

Christoph Spengler, Elmar Platzgummer, Hans Loeschner
IMS Nanofabrication GmbH (Austria)

Proc. SPIE 10810, 108100I (2018)

Online Publication Date: 10 October 2018

Withdrawn from Publication: 31 October 2018

Conference Date: 17-20 September 2018

Conference Location: Monterey, California, United States

Conference Title: Photomask Technology

Conference Chairs: Emily E. Gallagher, Jed H. Rankin

Publisher's Note: This conference presentation, originally published on 10 October 2018, was withdrawn per author request. 